\title{
Clinical Application of Omics Technology in the History of Primary Lactose Intolerance
}

\section{Ohood Alharbi}

Department of Nutritional Sciences, University of Toronto

Primary lactose intolerance (PLI) refers to a group of symptoms associated with lactose maldigestion, which arise due to a physiological decline in the activity of the enzyme lactase after weaning (1). Although Hippocrates was the first to describe the symptoms of PLI, it took 2000 years to recognize that these symptoms are due to an intolerance of lactose, which is the main sugar in milk (1). During the early 1970s, it was discovered that PLI is an inherited trait (1); however, the genetics behind the hereditary nature of PLI have puzzled many scientists. Due to the rapid development of "omics" technologies, from the 1990s until the present date, researchers have made strides toward understanding the genetic and epigenetic factors of PLI (1-4). The use of omics technology has helped to both identify PLI genotypes and determine its true prevalence in populations. Furthermore, many nutrigenomic and personalized nutrition studies use PLI gene variants to examine the association between dairy and disease risk. Results from these types of studies help in developing personalized dietary recommendations tailored to one's genotype to enhance health outcomes (5).

The early 1990s saw the discovery of state of the art omics technology, which led to the identification of the lactase gene. Despite this, the lactase gene is not associated with PLI prevalence around the world. In 2002, PLI genotypes were identified using another sophisticated omics technique that matched the history of PLI in nine families with a map of gene variants surrounding the lactase gene (4). These genotypes were associated with lactase activity levels, PLI symptoms, and dairy intake (6). This turning point in the history of PLI allowed for quantification of the true prevalence of PLI around the world (6). It also confirmed that PLI is our ancestors' trait, and that the ability to consume milk in adulthood is an acquired genetic trait (1). Furthermore, this discovery provided a new tool to differentiate between PLI and secondary lactose intolerance (1).

Genotypes of PLI are associated with different activ- ity levels of the enzyme lactase (4). However, based on the sucrase-lactase ratio, these genotypes were grouped into two traits: PLI and lactase persistence (LP; the ability to consume milk in adulthood) (7). In 2016, data from the Toronto Nutrigenomics and Health Study showed that those who possessed a genotype associated with intermediate lactase activity levels also had lower dairy intake and high risk of suboptimal vitamin $D$ concentrations (8). These findings were similar to the association observed in individuals with the PLI genotype, but to a lesser extent. Inadequate vitamin D intake has been a main concern associated with the dairy-free diet followed by those with PLI (7). Since tests used in a clinical setting don't differentiate between PLI, intermediate PLI, or LP (9), these results support the use of PLI genetic information to diagnose intermediate PLI genotypes, and examine this group for risks associated with low dairy intake.

In the presence of low activity levels of the enzyme lactase, lactose is digested by colonic bacteria (1). Relatively few studies have looked at the effect of dairy consumption on colonic bacteria in individuals with PLI. This is mainly due to the burden of symptoms associated with dairy consumption in these individuals, and the limited capability of techniques that can examine the effect of dairy on the broad spectrum of colonic bacteria (1). This was no longer an issue after the development of omics techniques, which allowed for measurement of changes in the thousands of bacterial species that reside in the colon. Using these techniques in a clinical trial, it was determined that lactose consumption in individuals with PLI led to an increase in the abundance of 4 types of good colonic bacteria, a decreased lactose intolerance symptom score, and increased tolerance to milk (10). This is in agreement with one of the dietary recommendations outlined for individuals with lactose intolerance, which encourages those with PLI to continue consuming dairy in small amounts to increase milk tolerance (7). Recently, using multiple "Omics" technologies, multiple 
factors were found to play a role in the epigenetics of PLI and LP, including dairy ingestion, age, and ethnicity $(2,3)$. This is in agreement with the "gene-culture co-evolution" theory, which states that LP is a result of a genetic changes introduced after humans adopted dairy as part of their diet $(1,6)$.

Developments in omics technologies have played a key role in understanding the effect of PLI genotypes on dairy consumption, as well as identifying intermediate PLI trait. Furthermore, omics technologies are currently being used to examine the effect of lactose intake in individuals with PLI. Future omics research examining PLI might develop promising personalized dietary recommendations that aim to help in decreasing PLI symptoms, as well as increasing milk tolerance and intake in those with PLI and intermediate PLI. These recommendations will also help both groups to consume the recommended number of servings of dairy per day.

\section{References}

1. Sahi T. Hypolactasia and lactase persistence. Historical review and the terminology. Scand J Gastroenterol Suppl. 1994;202:1-6.

2. Oh E, Jeremian R, Oh G, Groot D, Susic M, Lee K, et al. Transcriptional heterogeneity in the lactase gene within cell-type is linked to the epigenome. Sci Rep. 2017;7:41843.

3. Labrie V, Buske OJ, Oh E, Jeremian R, Ptak C, Gasiunas G, et al. Lactase nonpersistence is directed by DNA-variation-dependent epigenetic aging. Nat Struct Mol Biol. 2016;23(6):566-573.

4. Enattah NS, Sahi T, Savilahti E, Terwilliger JD, Peltonen L, Jarvela I. Identification of a variant associated with adult-type hypolactasia. Nat Genet. 2002;30(2):233-237.

5. Fenech M, El-Sohemy A, Cahill L, Ferguson LR, French TA, Tai ES, et al. Nutrigenetics and nutrigenomics: viewpoints on the current status and applications in nutrition research and practice. J Nutrigenet Nutrigenomics. 2011;4(2):69-89.

6. Ingram CJ, Mulcare CA, Itan Y, Thomas MG, Swallow DM. Lactose digestion and the evolutionary genetics of lactase persistence. Hum Genet. 2009;124(6):579-591.

7. Wilt TJ, Shaukat A, Shamliyan T, Taylor BC, MacDonald R, Tacklind J, et al. Lactose intolerance and health. Evid Rep Technol Assess (Full Rep). 2010(192):1-410.

8. Alharbi O, El-Sohemy A. LCT gene variant $-13910 \mathrm{C}>\mathrm{T}$, dairy in- take, and 25-hydroxyvitamin D plasma levels: a mendelian randomization study. FASEB J. 2016;30(1 Supplement):6-127.

9. Haberkorn BC, Ermens AA, Koeken A, Cobbaert CM, van Guldener C. Improving diagnosis of adult-type hypolactasia in patients with abdominal complaints. Clin Chem Lab Med. 2012;50(1):119-23.

10. Azcarate-Peril MA, Ritter AJ, Savaiano D, Monteagudo-Mera A, Anderson C, Magness ST, et al. Impact of short-chain galactooligosaccharides on the gut microbiome of lactose-intolerant individuals. Proc Natl Acad Sci USA. 2017;114(3):e367-e375.

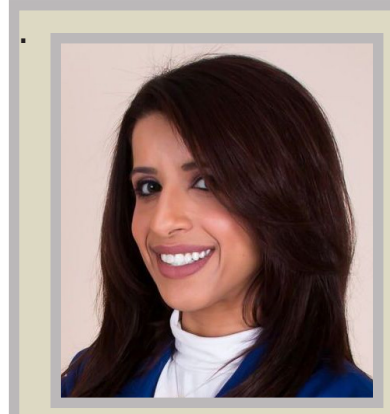

\section{Ohood Alharbi}

Ohood is a PhD candidate in the Department of Nutritional Sciences at the University of Toronto. Ohood completed an undergraduate degree in Medical Technology, specializing in microbiology and molecular biology. She pursued a graduate degree in Food and Nutrition in order to explore the relationship between diet and genetics. Her research interests include examining the interaction between diet, microbiome, and the human genome. Ohood is also an advocate for personalized nutrition for healthy living and well-being. 